\title{
Accelerated Ulcer Healing and Resistance to Ulcer Recurrence with Gastroprotectants in Rat Model of Acetic Acid-induced Gastric Ulcer
}

\author{
Tae Young Oh${ }^{1}$, Byung Ok Ahn¹, Eun Jung Jang², Joo Sang Park², Sang Jong Park², \\ Hyun Wook Baik ${ }^{2}$, and Ki-Baik Hahm²,* \\ ${ }^{1}$ Dong A Pharmaceutical Research Institute, Yongin 130-708, Korea \\ ${ }^{2}$ Digestive Disease Center, Daejin Medical Center Jesaeng Hospital at Bundang, Seongnam 463-774, Korea
}

Received 5 October, 2006; Accepted 26 October, 2006

\begin{abstract}
Summary Quality of ulcer healing (QOUH) is defined as ideal ulcer healing featuring with the fine granular ulcer scar, high functional restoration and the resistance to recurrence. This study was designed to compare the rates of QOUH achievement in rat gastric ulcer model between acid suppressant treated group and gastroprotectant treated group accompanied with elucidations of molecular mechanisms. Serosal injection of acetic acids for generating gastric ulcer and intraperitoneal (ip) injection of recombinant interleukin 1-beta (IL-1ß) for recurring healed ulcer was done in SD rats. The 72 rats were divided into three groups according to treatment as follows; Group I, no further treatment, Group II, 8 weeks treatment of

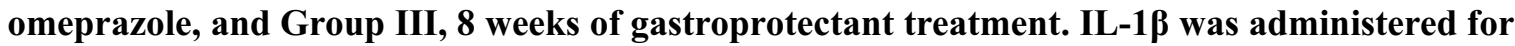
ulcer recurrence after 28 weeks of acetic acid injection. At four weeks after gastric ulcerogenesis, $58.3 \%$ (7/12) of active gastric ulcer were converted to healing stage in Group III, but $16.7 \%(2 / 12)$ in Group II and none in Group I, for which significant levels of epidermal growth factor, mucin, and pS2/trefoil peptide1 were contributive to these accelerated healings of Group III. ip injections of rIL-1 $(200 \mu \mathrm{g} / \mathrm{kg})$ at 28 weeks after acetic acid injection led to $100 \%$ of ulcer recurrence in Group I and $75.0 \%$ in Group II, but only $16.7 \%$ of Group III rats showed ulcer recurrence. Significantly attenuated levels of inflammatory cytokines including IL-2, transforming growth factor-alpha (TNF- $\alpha$ ), cyclooxygenase-2 (COX-2), nitrotyrosine were responsible for the resistance to ulcer recurrence in Group III. Conclusively, gastroprotectant might be prerequisite in order to achieve ideal QOUH through significant inductions of remodeling.
\end{abstract}

Key Words: QOUH, ulcer recurrence, ideal ulcer healing, gastroprotectant, remodeling

\section{Introduction}

Since the discovery of hydrochloric acid and pepsin in the stomach, the question that "Why does the stomach not digest itself?", "How does the gastric mucosa preserve its normal

\footnotetext{
*To whom correspondence should be addressed

Tel: +82-31-779-0393 Fax: +82-31-779-5079

E-mail: hahmkb@hotmail.com
}

integrity against noxious materials that are secreted?", and "How does the stomach resist against damaging Helicobacter pylori (H. pylori) or non-steroidal anti-inflammatory drugs (NSAIDs)?" has persisted [1-3], after which the identification of the presence of "gastric mucosal barrier" was imposed [4]. When the barrier is broken by the aggressive offensive factors, the gastric mucosa allows a back diffusion of gastric acid into the mucosal layers, leading to mucosal damages. Therefore, acid suppressants had been gold standard in the treatment of gastric ulcer with consider- 
able rates of healing and achievements of symptoms relief in accordance with the eradication of H. pylori, if associated $[5,6]$.

However, because the considerable portion of patients suffered from recurrence or complication in spite of maintenance medications or even after complete resolution [7], many investigators had performed diverse studies revealing the significance of gastric defense system to overcome these limitations of acid suppressant treatment. Several kinds of gastroprotectants are available in clinic, including sucralfate, rebamipide, misoprostol, ecabet sodium, sofalcone, DA-9601 (ethanol extracts of Artemisia asiatica), etc., achieving the several lines of clinical efficacy [8-11].

Ulcer healing is a dynamic process of filling the mucosal defect with epithelial and connective tissue cells, for which complex biological responses including cell proliferation, migration, differentiation, regeneration, active angiogenesis, and extracellular matrix deposition are required $[6,7,12$, 13]. Biological molecular processes for ulcer healing are comprised of inflammation, cell migration, mitosis, and remodeling, all of which are associated with the active synthesis and release of several peptide growth factors at the site of injury such as epidermal growth factor (EGF), transforming growth factor-alpha (TGF- $\alpha$ ), TGF- $\beta$, fibroblast growth factor (FGF) as well as trefoil peptide [14, 15]. The complex interaction of these proteins led to rapid restitution process composed of re-epithelialization, restoration of muscular components, angiogenesis, and tissue remodeling $[16,17]$.

The achievement of quality of ulcer healing (QOUH), ideal ulcer healing, is thought to be prostaglandin (PG)dependent because exogenous PGs could reverse events involved in ulcer recurrence, inflammatory response, retarded ulcer healing, and defective angiogenesis [18]. Even though, in clinic, chromoscopy is able to detect and differentiate whether the pattern of the gastric ulcer is flat on nodular [17, 19, 20], still there was limited report clearly showing the objective rate of QOUH achievement after either acid suppressant or gastroprotectant treatment, more scarse in elucidating the underlying molecular mechansism. Our study was designed to compare the healing efficiency and the resistance of ulcer recurrence between acid suppressant and gastroprotectant treatment in rat model of acetic acidinduced gastric ulcer and explored the underlying molecular mechanisms to explain the difference according to treatment plan. Omeprazole was administered as acid suppressant and DA-9601 was used as gastric cytoprotectant. DA-9601 used in the current study was demonstrated to possess cytoprotective actions in various experimental models, including ethanol-induced gastric mucosal damage, reflux esophagitis, and trinitrobenzosulfonic acid (TNBS)-induced colitis, of which mechanisms were stimulation of mucus, PG, glutathione and antioxidative actions [11, 21-24].

\section{Materials and Methods}

\section{Animals}

Sprague-Dawley rats with a body weight of $200 \mathrm{~g}-250 \mathrm{~g}$ were used in the experiments. Total 72 SD rats were used for the current experiment and were fed standard laboratory chow and tap water and kept in the room with controlled temperature $\left(22 \pm 1^{\circ} \mathrm{C}\right)$, humidity $(50 \%)$, and light cycle ( $12 \mathrm{~h}$ light $/ 12 \mathrm{~h}$ dark). The rats were fasted for $18 \mathrm{~h}$ before injection of acetic acid to the stomach. During fasting, all animals were kept in mesh-bottom cages to prevent coprophagy.

\section{Induction of acetic acid-induced gastric ulcer}

Under the anesthesia with ketamine, the laparotomy was done for acetic acid ( $40 \mu \mathrm{l}, 10 \% \mathrm{vol} / \mathrm{vol})$, which was injected to the subserosa of the stomach with micro-syringe to induce gastric ulcer. We tried to induce gastric ulcer in the same area of each stomach in the mid-fundus between the two branches of the main arterial vessel. The abdomen was sutured and the rats were returned to their cages. After operation, the rats were fasted for $48 \mathrm{~h}$ more with the supply of intravenous fluid infusion through carotid cannulation catheter and were fed normally thereafter (Fig. 1). To determine the size of ulcers and stages after 4 weeks or 24 weeks after acetic acid injection, animals were killed with an overdose of ether and their stomachs were removed. The stomachs were opened along the greater curvature and rinsed with cold saline. Then the stomachs were pinned to a cork board and then inflated, and the areas $\left(\mathrm{mm}^{2}\right)$ of the ulcers were determined under a dissecting macroscope (Olympus, Tokyo, Japan) and the classification of ulcer stage was determined based on the classification of Sakita and Miwa [25] as follows; A stage (active stage, $\mathrm{A}_{1}$, the surrounding mucosa is edematously swollen and no regenerating epithelium is seen. $A_{2}$, the surrounding edema has decreased, the ulcer margin is clear, and slight amount of regenerating epithelium is seen in the ulcer margin), $\mathrm{H}$ stage (healing stage, $\mathrm{H}_{1}$, the white coating is becoming thin and the regenerating epithelium is expanding into the ulcer base. The gradient between ulcer margin and the ulcer floor is becoming flat. The diameter of the mucosal defect is about one half to two thirds that of $\mathrm{A}_{1} ; \mathrm{H}_{2}$, the defect is smaller than in $\mathrm{H}_{1}$ and the regenerating epithelium covers most of ulcer floors and the area of white coating is about a quarter to one third that of $A_{1}$ ) and $S$ stage (scarring stage, $S$, the regenerating epithelium completely covers the floor of ulcer. The white coating has disappeared. Upon close observation, many capillaries can be seen, which is called "red scar" $\left(\mathrm{S}_{1}\right)$, which become white scar with the reduction of redness, called "white scar" $\left(\mathrm{S}_{2}\right)$. 


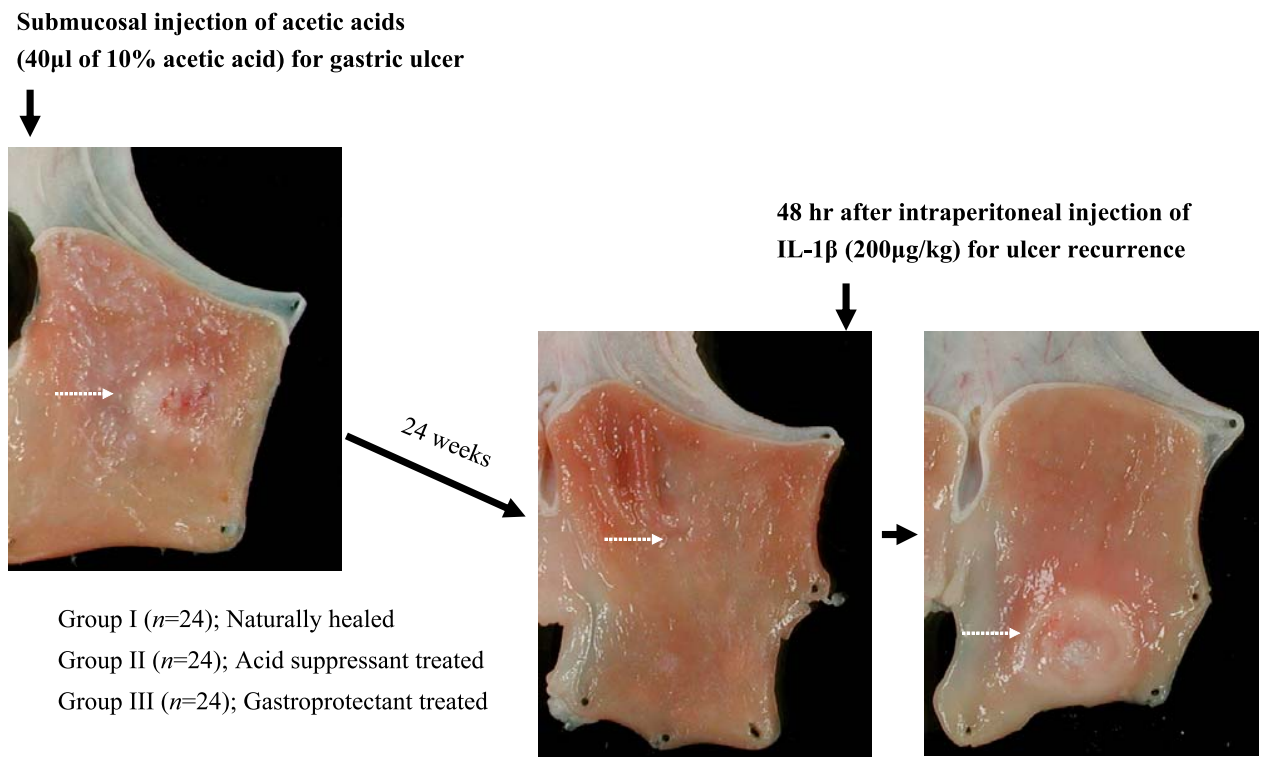

Fig. 1. Experimental protocol for ulcer healing and ulcer recurrence according to group. Group I denotes the group of rats healed naturally up to 28 weeks after serosal injection of acetic acid, Group II denotes the group of rats treated with 8 weeks of proton pump inhibitor, intraperitoneal injections of omeprazole, and Group III denotes the group of rats receiving 8 weeks of gastroprotectant, DA-9601 in pellet diets. After 28 weeks, all rats were administered with intraperitoneal injections of $200 \mu \mathrm{g} /$ $\mathrm{kg}$ recombinant IL-1 $\beta$ irrespective of group and sacrificed after $48 \mathrm{~h}$ later to observe ulcer recurrence. Meanwhile, 12 rats in each group were killed at 4 weeks after acetic acid injection and the remaining 12 rats in each group were sacrificed at 28 weeks after acetic acid injection. Preliminary study confirmed that 28 weeks of duration were enough for complete ulcer healing even without any treatment. The classification of ulcer stage was determined based on the classification of Sakita and Miwa [25] as A (active) stage, $\mathrm{H}$ (healing) stage, and S (scarring) stage.

\section{Experimental group assignment}

The 72 rats were grouped in to three as follows; Group I $(n=24)$ is rats without any treatment at all, natural healing group, Group II $(n=24)$ is rats treated with omeprazole $10 \mathrm{mg} / \mathrm{kg}$ intaperitoneally for 8 weeks, and Group III $(n=24)$ is rats treated with diet pellet containing $100 \mathrm{mg} / \mathrm{kg}$ of DA-9601, which is ethanol extract of Artemisia asiatica showing anti-inflammatory, antioxidative, and PG inducing actions. Half of these rats in each group were killed at 4 weeks after acetic acid injection and the remaining 36 rats were sacrificed at 28 weeks 2 days after interleukin-1 $\beta$ (IL-1 $\beta$ ) injection.

Gastric ulcer recurrence with intraperitoneal injection of recombinant $I L-1 \beta$

As a preliminary study to document that 28 weeks was enough for natural healing after acetic acid induced gastric ulcers, twelve rats with naturally healed ulcers were killed before the administration of recombinant human IL-1 $\beta$ (Genzyme, Boston, MA) to observe the ulcer stage. Since all ulcers were found to heal completely at 28 weeks after ulcer induction with acetic acid, twelve animals in each group were given recombinant human IL-1 $\beta(200 \mu \mathrm{g} / \mathrm{kg})$ intraperitoneally according to Watanabe's model of ulcer recurrence [26]. Another preliminary study was done to document the best time point for ulcer recurrence and we obtained the result that at $48 \mathrm{~h}$ after IL-1 $\beta$ injection $100 \%$ of ulcers were recurred, whereas $66.7 \%$ ulcer recurred after $24 \mathrm{~h}$ of IL-1 $\beta$ injection in our model. Therefore, the animals were killed $48 \mathrm{~h}$ later to evaluate the rate of ulcer recurrence according to treatment group (Fig. 1). Ulcer specimens were fixed in $10 \%$ buffered formalin or immediately frozen in liquid nitrogen for hematoxylin-eosin (H\&E) staining and further immunohistochemical staining. Tissues were excised at ulcer margin and immediately frozen for elucidating their molecular mechanisms.

Western blot for COX-2, TGF- $\alpha, E G F$ and $p S 2 / T F F 1$ in gastric homogenates of gastric ulcers

Pooled gastric mucosa was homogenized in ice-cold $20 \mathrm{mM}$ Tris- $\mathrm{HCl}$ buffer, pH 7.5 containing $2 \mathrm{mM}$ EDTA, $0.5 \mathrm{mM}$ EGTA, $300 \mathrm{mM}$ sucrose, $2 \mathrm{mM}$ phenylmethyl sulfonyl fluoride (PMSF) with a tissue homogenator at $4^{\circ} \mathrm{C}$. Ten microgram of the protein was subjected to electrophoresis on an $8 \%$ SDS-PAGE gel and transferred onto PVDF membrane. The blots were blocked with 5\% nonfat dry milk, and then incubated overnight at $4{ }^{\circ} \mathrm{C}$ with primary antibodies for HSP 27, HSP 70, or $\alpha$-tubulin (Santa cruz Biotech, Santa Cruz, CA), followed by probing with 1:2000 diluted HRP-conjugated secondary antibody. The immuno- 
complex were detected using ECL detection kit (Ammersham Phamacia Biotec, Uppsala, Sweden). cyclooxygenase2 (COX-2), TGF- $\alpha$, EGF, trefoil peptide (pS2/TFF1), platelet derived growth factor (PDGF) and vascular endothelial growth factor (VEGF) were measured.

\section{Immunofluorescence staining and Alcian blue staining}

The frozen sections were fixed in cold acetone at $-20^{\circ} \mathrm{C}$ for $5 \mathrm{~min}$ and incubated overnight with each 1:100 diluted antibody, including pS2, EGF, all purchased from Santa Cruz Biotech. After washing with phosphate-buffered saline, they were incubated for $30 \mathrm{~min}$ with fluorescein isothiocyanate (FITC)-conjugated anti-rabbit immunoglobulin (Sigma Chemical Co., Saint Louis, MO) diluted 1:100. Immuno-fluorescence signal was evaluated using a fluorescence microscope. EGF, TFF1 and mucin were determined

\section{RT-PCR analysis}

Total RNA was isolated from cells with appropriate treatment using the TRIzol reagent (Life technologies, Milan, Italy) and $2 \mu \mathrm{g}$ of total RNA was reverse transcribed according to the manufacturers' instructions of M-MLV Reverse transcriptase (Promega, Madison, WI). The PCR was performed by using the Premix Ex Taq kit (Takara, Chiba, Japan) with specific primers as follows: 5'-GAA GTG TAT TAG GAC GCT GG-3' and 5'-TGT AGA GGC TGT CCA $A G T C T-3^{\prime}$ for PDGF, 5'-GTC GTA CGA TGG GTA CTG $C C-3^{\prime}$ and 5'-GCG CAG CTT CCA CCA ACG TA-3' for EGF, 5'-TGC ACC CAC GAC AGA AGG GGA-3' and 5'TCA CCG CCT TGG CTT GTC ACA T-3' for VEGF, 5'-TGA AGG TCG GTG TCA ACG GAT TTG GC-3' and 5'-CAT GTA GGC CAT GAG GTC CAC CAC-3' for GAPDH.

\section{Enzyme-linked immunosorbent assay for cytokines}

Immunoreactive cytokine level of IL-2, tumor necrosis factor-alpha (TNF- $\alpha$ ), and IL-10 was measured in gastric mucosa of rats by using enzyme-linked immunosorbent assay (ELISA) kits purchased from R\&D Systems Europe (Abington, U.K.).

\section{Histological examination for quality of ulcer healing}

Ulcer specimens were fixed in $10 \%$ buffered formalin and embedded in paraffin. Tissues were sectioned in $4 \mu \mathrm{m}$ thickness for H\&E staining. Neutral glycoproteins (mucin) were stained by periodic acid-Schiff. We evaluated the quality of ulcer healing based on the histological assessment of epithelial re-consititution 6 months after treatment. Poor quality of ulcer healing was defined as histological abnormalities included dilated gastric glands and pits, reduced height of granulation tissue and inadequate secretion of neutral mucin.

Vol. 42, No. 3, 2008

\section{Statistical analysis}

Results are expressed as the mean $\pm \mathrm{SD}$. The data were analyzed by one-way analysis of variance (ANOVA), and the statistical significance between groups was determined by Duncan's multiple range test. Statistical significance was accepted with a $p<0.05$.

\section{Results}

Accelerated healing of acetic acids-induced gastric ulcer in rats treated with gastroprotectant than acid suppressant at 4 weeks after gastric ulcerogenesis

To determine the size and staging of gastric ulcers after each treatment, animals were killed with an overdose of ether and their stomachs were removed. Then the stomachs were pinned to a cork board and then inflated, and the areas $\left(\mathrm{mm}^{2}\right)$ of the ulcers were determined and the classification of ulcer stage was determined based on the classification of Sakita and Miwa [25]. Twelve rats in each group were sacrificed at 4 weeks after intragastric acetic acid injection for assessing the healing status according to group. All gastric ulcers in Group I and 10 out of 12 in Group II were still in active stage of gastric ulcer (A stage) at 4 weeks after acetic acid injections, but, 7 out of 12 rats (58.3\%) in Group III were already in healing stage of gastric ulcer, mostly $\mathrm{H}_{2}$ stage ulcer. As seen in Fig. 2, the ulcer niche was considerably decreased in Group III, of which ulcer base was almost disappeared reaching to $\mathrm{S}$ stage of ulcer in some rats, suggesting that gastroprotectant treatment imposed rather accelerated healing process (Fig. 2).

The pooled expressions of TGF- $\alpha$ were significantly increased in both Group II and III than Group I. The expressions of EGF were considerably increased only in Group III. Though there was no significant difference in the expression of either COX-2 or pS2/TFF1 with western blot analysis (Fig. 4A), the immunohistochemical stainings of EGF and pS2/TFF1 were significantly increased in Group III. In detail, prominent gastric glands showing high expressions of $\mathrm{pS} 2$ /TFF1 observed in Group III were UACL (ulcer associated cell lineage), precursor for regenerating gastric glands (Fig. 4B). The expression of neutral mucin with Alcian blue staining was apparently increased in drugtreated groups (Group II and Group II) than naturally healing group (Fig. 4C).

\section{Significant resistance to ulcer recurrence in group III,} treated with gastroprotectant

Since preliminary study showed that $100 \%$ of gastric ulcers induced by acetic acid injection was healed completely after 24-28 weeks, converting into S stage of gastric ulcer and ulcer was recurred approximately 75\% after 24 and $100 \%$ after $48 \mathrm{~h}$ of intraperitoneal injections of $200 \mu \mathrm{g} /$ $\mathrm{kg}$ of IL-1 $\beta$, we determined the point of ulcer recurrence 


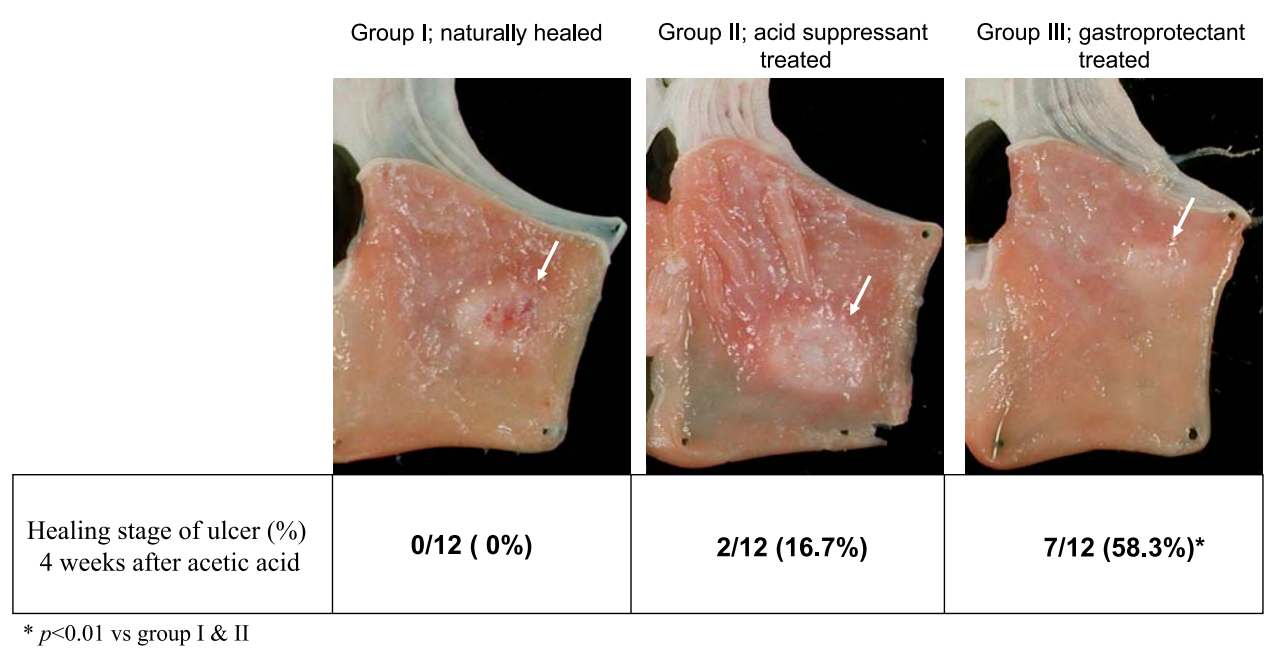

Fig. 2. Gastric ulcer stage assessed at 4 weeks after acetic acid according to group. All gastric ulcers of group I and 10 out of 12 in Group II were still active stage of gastric ulcer, telling that two cases of Group II was healing stage, whereas 7 out of 12 rats $(58.3 \%)$ in Group III were found to be healing stage ulcer, mostly $\mathrm{H}_{2}$ stage ulcer. Accelerated healing of gastric ulcer was noted in group treated with gastroprotectant.

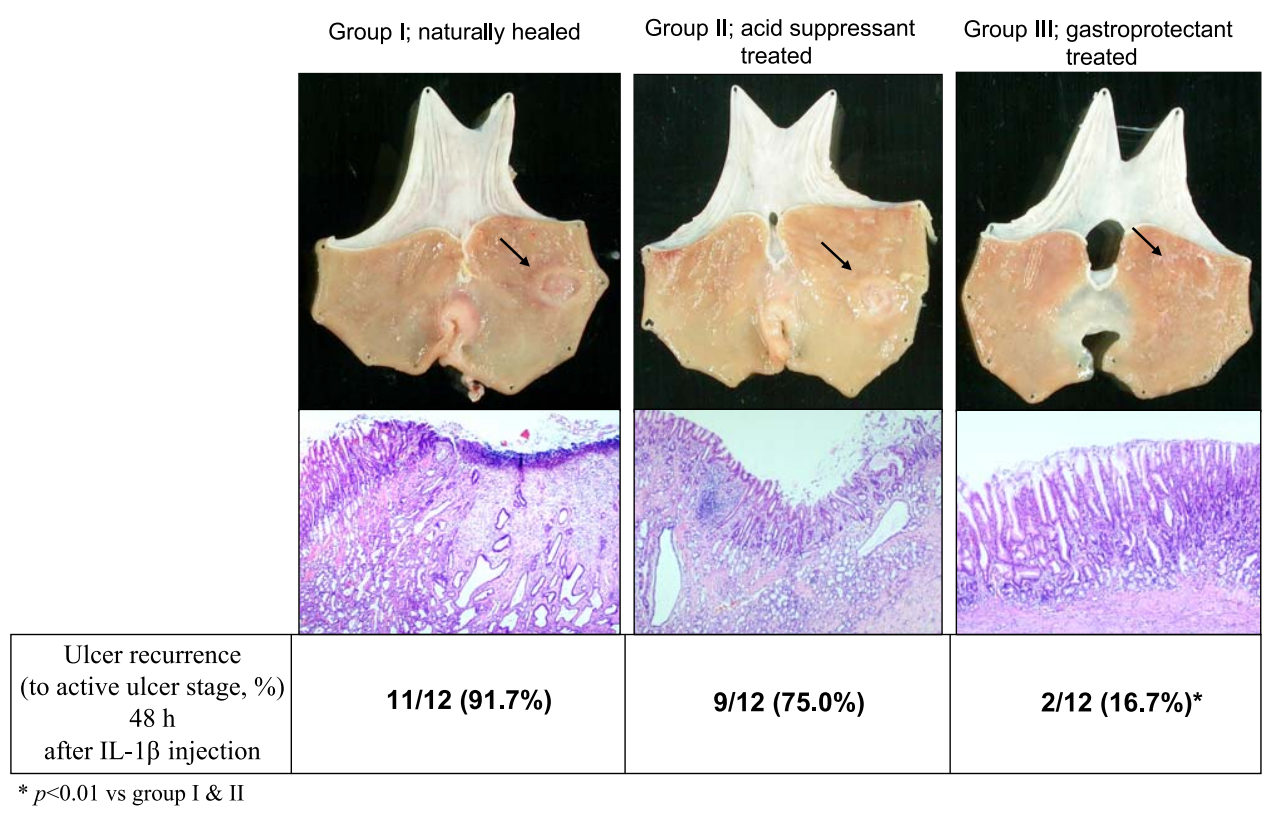

Fig. 3. Ulcer recurrence rates after IL-1 $\beta$ administration according to groups. Since preliminary study showed that $100 \%$ healing of gastric ulcer was noted after 24-28 weeks, converting into S stage of gastric ulcer, and ulcer was recurred after $24-48 \mathrm{~h}$ of intraperitoneal injections of $200 \mu \mathrm{g} / \mathrm{kg}$ of IL- $1 \beta$, we administered IL- $1 \beta$ to all rats of experimental groups and sacrificed $48 \mathrm{~h}$ later. 11 out of 12 rats with scarred gastric ulcer $(91.7 \%)$ in Group I were recurred after IL-1 $\beta$ injection and 9 out of 12 rats (75.0\%) showed ulcer recurrence in Group II. However, only 2 out of $12(16.7 \%)$ showed ulcer recurrence and the remaining 10 out of 12 rats remained intact even after the administration of IL-1 $\beta$, signifying that considerable resistance of ulcer recurrence was achieved in group treated with gastroprotectant.

experiment at 28 weeks after acetic acid and the point of sacrifice at $48 \mathrm{~h}$ later after IL-1 $\beta$ injection (Fig. 1).

11 out of 12 rats with scarred gastric ulcer $(91.7 \%)$ in Group I were recurred after IL-1 $\beta$ injection and 9 out of 12 rats $(75.0 \%)$ showed ulcer recurrence in Group II, all converting to A stage of ulcer after IL-1 $\beta$ injection. However, only 2 out of $12(16.7 \%)$ showed ulcer recurrence and the other 10 out of 12 rats remained in S stage gastric ulcer even after the administration of intraperitoneal IL- $1 \beta$, signifying that considerable resistance of ulcer recurrence 
(A)

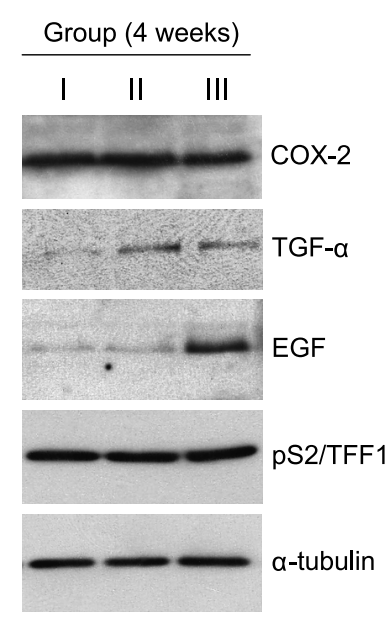

(C)
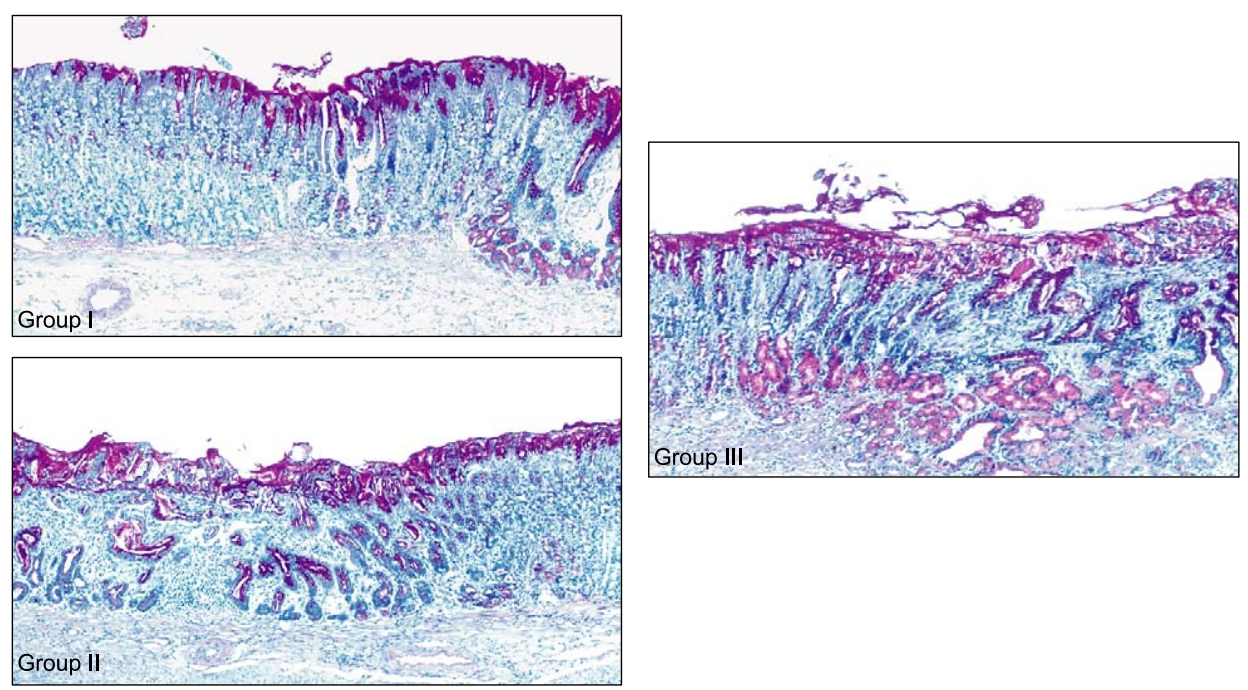

pS2/TFF1
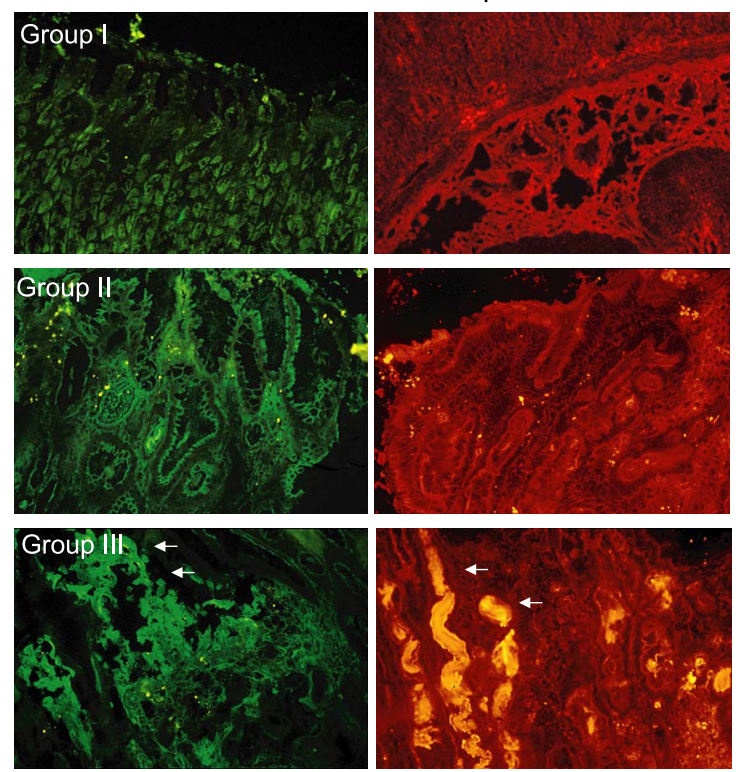

Fig. 4. Western blot of COX-2, TGF- $\alpha$, EGF, and pS2/TFF1 and immunohistochemical staining of EGF and pS2/TFF1 according to group (A) Western blot. The pooled expressions of TGF- $\alpha$ were significantly increased in Group II and III than group I and the expressions of EGF were considerably increased in Group III. There was no significant difference in the expression of either COX-2 or pS2/TFF1. (B) Immunofluoroscence staining for EGF and pS2/TFF1 at ulcer margin. Immunohistochemical stainings of EGF and pS2/TFF1 were significantly increased in Group III. Prominent gastric glands showing high expressions of pS2/TFF 1 observed in group III were UACL (ulcer associated cell lineage) $(\times 200$ magnification). (C) The change of neutral mucins at the periphery of acetic acids-induced gastric ulcer with Alcian blue mucin staining. The expression of neutral mucin with Alcian blue mucin staining was apparently increased in drug-treated groups (Group II and Group II) than natural healing group $(\times 100$ magnification).

was achieved in group treated with gastroprotectant, DA9601 (Fig. 3). Besides of DA-9601, similar resistance to ulcer recurrence was shown in rats group treated with other kinds of gastroprotectants like rebamipide and ecabet sodium; the overall rates of resistance to ulcer recurrence experiment according to kind of gastroprotectants were $2 / 12$
(16.7\%) with DA-9601, 3/12 (25.0\%) with rebamipide, and $3 / 11(27.2 \%)$ with ecabet sodium, respectively.

Histopathological observations before the induction of ulcer recurrence with IL-1 $\beta$ were quite differed according to group as seen in Fig. 5, even though all their gross morphology looks like scarred ulcer. Dilated and distorted 

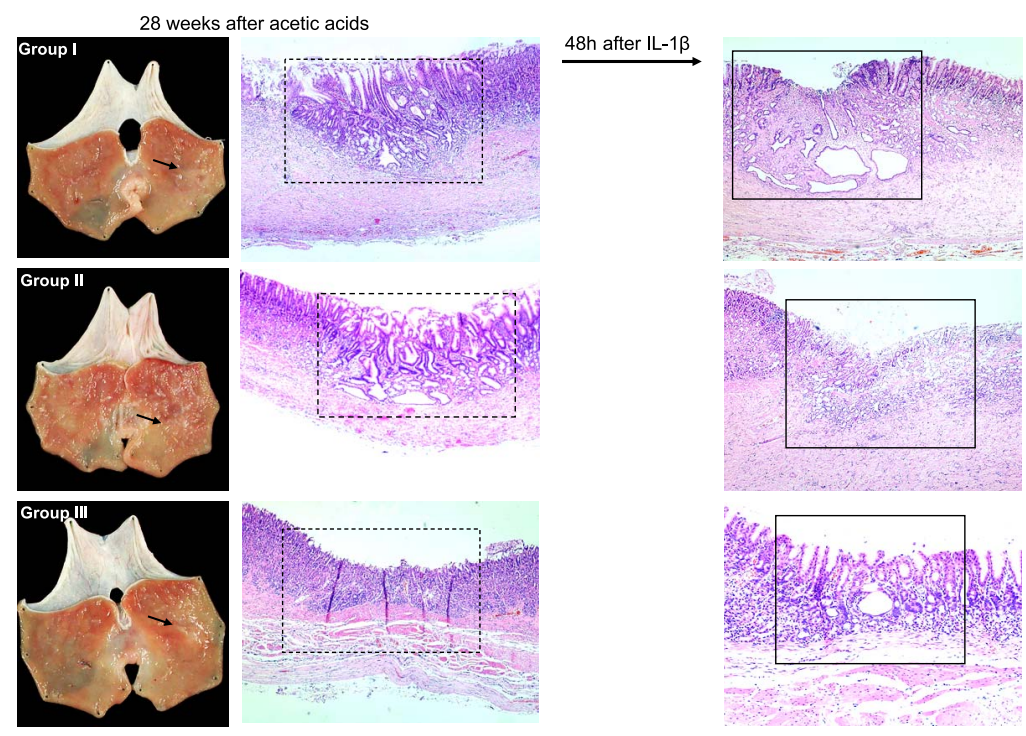

Fig. 5. Histopathology of scarred gastric ulcer before and after IL-1 $\beta$ injection according to treatment. Gross observation before IL-1 $\beta$ injection at 28 weeks after acetic acid induced gastric ulcer showed that all acetic acids-induced gastric ulcers become scarring stage ( $\mathrm{S}_{1}$ or $\mathrm{S}_{2}$ stage) irrespective of group. However, histopathological observations were differed so much according to group. Dilated and distorted gastric glands were noted in both Group I (naturally healed) and Group II (PPI-treated group), whereas normal looking restored gastric mucosa was seen in Group III (DA-9601 treated group). Significant infiltrations of inflammatory cells and denuded mucosal layers were seen after IL-1 $\beta$ injection in Group I and II, but attenuated inflammatory response and conserved mucosal layer were seen in Group III, signifying that excellent remodelling of regenerated gastric mucosa, hard to discriminate between intact gastric mucosa and regenerated gastric mucosa, was achieved in rats treated with gastroprotectants and might be responsible for resistance to ulcer recurrence after IL-1 $\beta$ injection.

gastric glands were noted in both Group I (naturally healed) and Group II (proton pump inhibitor (PPI)-treated group), whereas normal looking, well restored and remodeled gastric mucosa was seen in Group III (DA-9601 as gastroprotectant-treated group). We defined the histological morphology of Group III after 28 weeks was remodeled regeneration. Significant infiltrations of inflammatory cells and denuded mucosal layers were seen after IL- $1 \beta$ injection in Group I and II, whereas considerably attenuated inflammatory response and conserved mucosal layer were seen in Group III even after IL-1 $\beta$ injection, signifying that excellent remodeling of regenerated gastric mucosa, hard to discriminate between intact gastric mucosa and regenerated gastric mucosa, was achieved in rats treated with gastroprotectants and might be responsible for resistance to ulcer recurrence (Fig. 5).

The molecular basis for the resistance to ulcer recurrence in rats treated with gastroprotectant

The mean levels of IL-2 were significantly lowered in Group III than Group I and Group II and the mean levels of TNF- $\alpha$ were significantly decreased only in Group III. However, there was no significant difference in IL-10 levels between groups. This result suggested that lesser degrees of gastric inflammation as seen in histopathology were provoked in rats treated with gastroprotectants, even though treated with potent inflammatory cytokine, IL-1 $\beta$ (Fig. 6A). Significantly attenuated expressions of either COX-2 or nitrotyrosine were noted in rats showing resistance to ulcer recurrence irrespective of treatment group, suggesting COX2 and nitrotyrosine might contribute to ulcer recurrence. The expressions of HSP 27 were increased in Group III, rats treated with DA-9601, suggesting the lowered recurrence of gastric ulcer after IL-1 $\beta$ injection might be partly related to the cytoprotective effects of molecular chaperone, HSP 27 (Fig. 6B). However, growth factors did not contribute to ulcer recurrence since no significant difference in the expressions of EGF, PDGF, and VEGF among either treatment groups or status of gastric ulcer was noted (Fig. 6C).

\section{Discussion}

Our current results showed that gastroprotectant was quite efficient in either accelerating the ulcer healing at the early phase of ulcer healing or hindering the recurrence of gastric ulcer after complete ulcer healing, whereas acid suppressant was somewhat inferior in these aspect of ulcer healing compared to gastroprotectant. Simply stated, the achievement of ideal QOUH could only be accomplished with intervention of the enhancement of gastric defense systems. Therefore, though acid suppressant is gold standard treatment for gastric ulcer, the anti-secretory agents alone were 


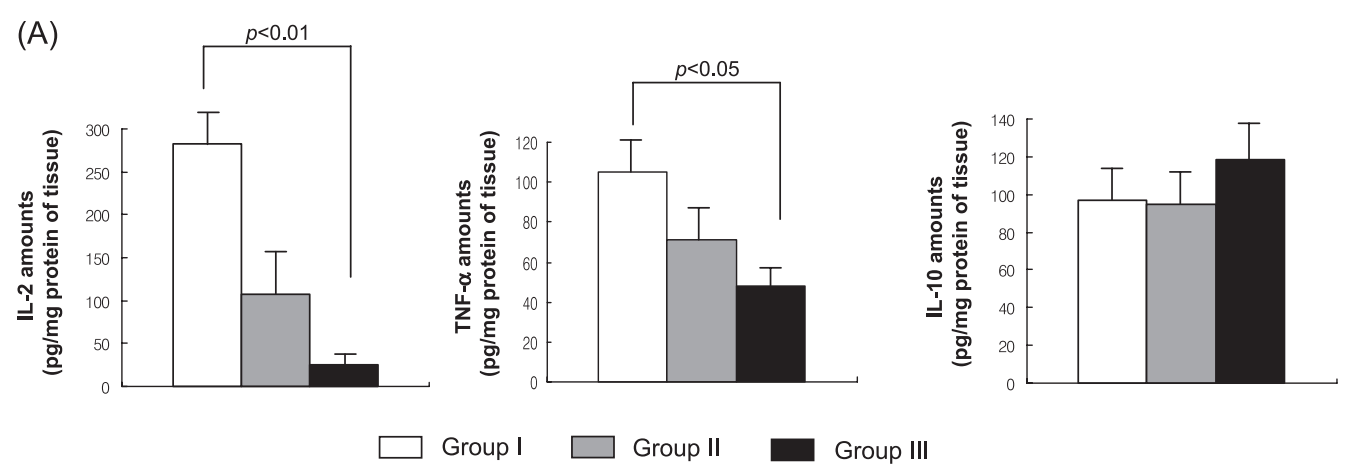

(B)

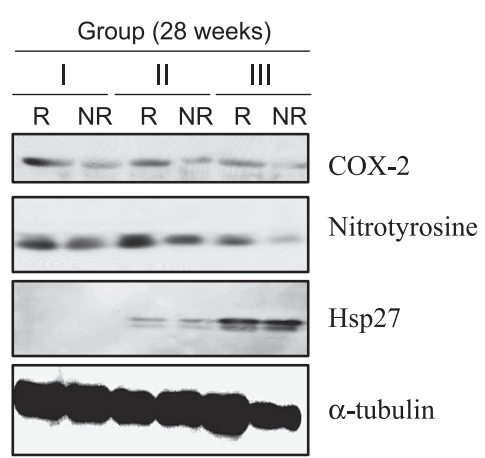

(C)

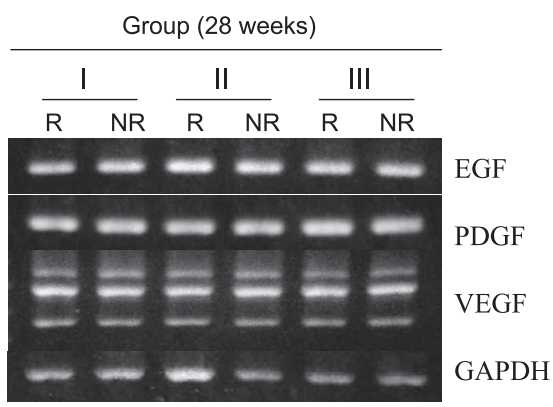

R: Ulcer recurrence after IL-1 $\beta$

NR: Resistance to ulcer recurrence after IL-1ß

Fig. 6. (A) The changes of mucosal IL-2, TNF- $\alpha$, and IL-10 according group. The mean levels of IL-2 were significantly lowered in Group III than Group I and II and the mean levels of TNF- $\alpha$ was significantly decreased only in Group III. However, there was no significant difference in IL-10 levels between groups. This result suggested that lesser degrees of gastric inflammation were provoked in rats treated with gastroprotectants. (B, C) Expressions of COX-2, nitrotyrosine, HSP 27, and growth factors according to group Western blot for COX-2, nitrotyrosine, and HSP27 according to group and status of gastric ulcer. Significantly attenuated expressions of either COX-2 or nitrotyrosine were noted in rats showing resistance to ulcer recurrence irrespective of treatment group, suggesting COX-2 and nitrotyrosine might contribute to ulcer recurrence. The expressions of HSP 27 were increased in Group III, rats treated with DA-9601, suggesting the lowered recurrence of gastric ulcer after IL-1 $\beta$ injection might be related to the cytoprotective effects of molecular chaperone, HSP 27. (C) RT-PCR for EGF, PDGF, and VEGF according group and status of gastric ulcer. No significant difference in the expressions of EGF, PDGF, and VEGF among either treatment groups or status of gastric ulcer.

not sufficient for reaching QOUH, necessitating the combined use of anti-secretory and gastroprotective agent. We also added the plausible molecular bases for QOUH achievement with DA-9601 treatment, that was, efficient remodeling of regenerated gastric mucosa, intervention of several growth factors, abundant gastric mucins, including trefoil proteins like pS2/TFF1, and significant suppressions of inflammatory cytokines like IL-2, TNF- $\alpha$, COX-2, and nitrosative stress.

Although we presented the detailed data about DA-9601 treatment, ethanol extracts of Artemisia asiatica [11], among several gastroprotectants $[8-10]$, which is one of gastroprotectants prescribed popularly for the treatment of gastritis and peptic ulcer disease in Korea, possessing actions like anti-inflammatory, cytoprotective, restorative, and antioxidative actions $[11,21-24]$, we repeated the same experi- ment with other gastroprotectants available in clinic like rebamipide and ecabet sodium, and found that the overall rates of resistance to ulcer recurrence experiment according to gastric cytoprotectants were $2 / 12(16.7 \%)$ with DA-9601, $3 / 12(25.0 \%)$ with rebamipide, and 3/11 (27.2\%) with ecabet sodium, respectively. Hence, we can't ignore the principal role of acid suppressant in the treatment of gastric ulcer, our suggestions from the current experiment were that in order to achieve rapid symptom relief, accelerated healing, and resistance to ulcer relapse, the combined use of acid suppressant and gastroprotectant should be considered. Therefore, we would like to stress our novel finding that gastroprotectant treatments were not merely supplementary in the treatment of gastric diseases, otherwise signifying that gastroprotectants might be essential and prerequisite for better healing. 
Since, by definition, an ulcer penetrates through the muscularis mucosa into the underlying submucosa and sometimes to the muscularis externae, repair of such damage requires the complete reestablishment of a connective tissue and reformation of glandular architecture [27]. The healing of ulcer is accomplished by filling of the mucosal defect through the regeneration of gastric glands and ECM to restore mucosal structure. Therefore, several growth factors have been implicated in this process by regulating cell functions, such as cell proliferation, migration, differentiation, secretion, and degradation of ECM since the exogenous administration of either natural or recombinant analogues of the growth factors is reported to accelerate the healing process [28]. Some examples of key growth factors involved in immediate ulcer repair are heparin, EGF, basic fibroblast growth factor (bFGF), TNF- $\alpha$, hepatocyte growth factor (HGF), TGF- $\alpha$, TGF- $\beta$, and trefoil peptide (TFF). Also, upregulation of receptors for some of these growth factors, such as EGF receptor, has been demonstrated to occur in the cells lining the ulcer margin. A new cell lineage can be observed at the ulcer margin that secretes EGF and TFF, so called ulcer associated cell lineage (UACL) [13, 29].

TFF is a group of small molecule polypeptide and mainly secreted by gastrointestinal mucous cells. There are three kinds of TFF found in mammals, which are stomach specific TFF (pS2/TFF1), spasmolytic polypeptide (SP/TFF2), and intestinal trefoil peptide (ITF/TFF3) [30], which has the function of mucosa protection, epithelial restitution, carcinoma suppression, and apoptosis adjustment [31]. Since the stimulus for increased epithelial cell proliferation in the mucosa of ulcer margin is most likely initiated by EGF and/or TGF- $\alpha$, the levels of EGF and/or TGF- $\alpha$ are closely correlated with accelerated healing. Immunohistochemical studies demonstrated that cells lining dilated gastric glands at the ulcer margin display an enormous increase in expression of EGF, EGFR, and TNF-a at the initial stage (1-7 days) after ulcer induction [7, 13, 15]. Moreover, the gastric ulceration can induce the development of a novel cell lineage, which grows from the bases of existing crypts or glands to form new glands, so called, ulcer associated cell lineage (UACL). All of these facts can explain how group III can achieve $58.3 \%$ of $\mathrm{H}$ stage gastric ulcer with even 4 weeks of gastroprotectant administration (Fig. 2).

In addition to accelerated ulcer healing, another implication of gastroprotectants in ulcer healing was significant attenuation of the gastric ulcer recurrence. A rat model of induction of gastric ulcer recurrence by IL-1 $\beta$ was first developed by Watanabe $\mathrm{T}$ et al. [32], which is useful for investigating the pathogenesis of gastric ulcer recurrence as approximately $80-100 \%$ of healed ulcer recurs at sites of scarred mucosa within $48 \mathrm{~h}$ after injection of IL-1 $\beta$. As seen in Fig. 4, the process of ulcer recurrence has been known to be neutrophil-dependent, oxidative stress depen- dent, and also gastric acid engaged. We could find the plausible mechanisms for resistance to ulcer recurrence in group III, which were excellent remodeling activity, enrichments of molecular chaperones like heat shock protein 27 (HSP27), significant decreases in inflammatory cytokines like COX-2, IL-2, and TNF- $\alpha$, and quenching nitrosative stress associated with the infiltrations of inflammatory cells after IL- $1 \beta$.

Heat shock proteins (HSPs) act as molecular chaperones and exhibit various functions, including protection against apoptosis, protease inhibition and cross-linkage to other proteins. While the induction of HSPs seems to be important for protection against apoptosis, protease inhibition, refolding, and activity of partially denatured proteins, other reports indicate that their beneficial effect may be especially important for the later phase of regeneration, when oxidative stress caused by infiltrating inflammatory cells may oppose tissue repair [33, 34], signifying that HSP27 induction might be one of mechanisms lower recurrence of gastric ulcer after IL-1 $\beta$ in gastroprotectant administration. COX, the key enzyme for synthesis of PG, exists in two forms (COX-1 and $\mathrm{COX}-2$ ). $\mathrm{COX}-1$ is constitutively expressed in the GI tract in large quantities and has been suggested to maintain mucosal integrity through continuous generation of PGs, COX-2 is induced predominantly during inflammation [35]. As seen in Fig. 5A, the levels of COX-2 were higher in recurrence rats than resistance to ulcer recurrence irrespective of groups, suggesting higher COX-2 levels might reflect the more gastric inflammations with profuse neutrophil infiltrations.

Besides of COX-2 expressions, nitrotyrosine has been used as a biological marker for the pathogenic consequences of endogenous exposure to nitric oxide-related compounds and reactive nitrogen species, reflecting the formation of peroxynitrite $\left(\mathrm{ONOO}^{-}\right)$, the oxidative damage of which may be assessed by the detection of nitrated [36] protein tyrosine residue (nitrotyrosine). Therefore, these specific markers carry the risk for either carcinogenesis risk or severe inflammatory response [37]. Gastroprotectant showed significant inhibitory activity against nitrotyrosine formation as seen in Fig. 6B.

Ulcer healing is a complicated process requiring interaction between ECM proteins and the granulation tissue supplying the connective tissue cells and endothelial cells and the quality of ulcer repair remains crucial for the stability of the injured tissue and for preventing recurrence. Therefore, remodeling may be critical for proper restoration of gastric glands and wall integrity [28, 38, 39]. Careful histopathologic inspections of regenerated gastric mucosa led to us find the very different histology of regenerated gastric glands between natural healing or acid suppressant treated groups and gastroprotectant treated group. The regenerated gastric glands were dilated, distorted, and variable sized gastric glands in acid suppressant group, but 
well maturated, organized, slender, and similar sized gastric glands in gastroprotectant treated group, so called achievement of remodeling during regenerations and restitution.

However, since gastric acid is required for recurrence of gastric ulcers caused by IL-1 $\beta$ [32], and gastric acid stimulates the inflammatory process in scarred mucosa during ulcer recurrence, we propose that QOUH could be efficiently achieved with treatment of both acid suppressant and gastroprotectant. Qualitative maturation with gastroprotectants in addition to quantitative achievement with acid suppressants is warranted for ideal and qualified ulcer healing. In conclusion, our data showed that gastroprotectant is not a merely supplementary in the treatment of gastric ulcer for enhancing defense factors, but prerequisite treatment for qualified ulcer healing.

\section{References}

[1] Tsukim, Y. and Okabe, S.: Recent advances in gastrointestinal pathophysiology: role of heat shock proteins in mucosal defense and ulcer healing. Biol. Pharm. Bull., 24, 19, 2001.

[2] Robert, A., Nezumis, J.E., and Phillips, J.P.: Inhibition of gastric secretion by prostaglandin. Am. J. Dig. Dis., 12, 1073-1076, 1967.

[3] Robert, A.: Cytoprotection by prostaglandins. Gastroenterology, 77, 761-767, 1979.

[4] Davenport, H.W.: Salicylate damage to the gastric mucosal barrier. N. Engl. J. Med., 8, 1307-1312, 1967.

[5] Allen, A.: Garner A. Mucus and bicarbonate secretion in the stomach and their possible role in mucosal protection. Gut, 21, 249-262, 1980.

[6] Wallace, J.L.: Mechanisms of protection and healing: current knowledge and future research. Am. J. Med., 110, 19S-23S, 2001.

[7] Jones, M.K., Tomikawa, M., Mohajer, B., and Tarnawski, A.S.: Gastrointestinal mucosal regeneration; role of prowth factors. Frontiers in Bioscience, 4, 303-309, 1999.

[8] Arakawa, T., Kobayashi, K., Yoshikawa, T., and Tarnawski, A.: Rebamipide, Overview of its mechanisms of action and efficacy in mucosal protection and ulcer healing. Dig. Dis. Sci., 43, 5S-13S, 1998.

[9] Murata, H., Kawano, S., Tsuji, S., Kamada, T., Matsuzawa, Y., Katsu, K., Inoue, K., Kobayashi, K., Mitsufuji, S., Bamba, T., Kawasaki, H., Kajiyama, G., Umegaki, E., Inoue, M., and Saito, I.: Combination therapy of ecobet sodium and cimetidine compared with cimetidine alone for gastric ulcer: prospective randomized multicenter study. J. Gastroenterol. Hepatol., 18, 1029-1033, 2003.

[10] Kabir, A.M., Shimizu, K., Aiba, Y., Igarashi, M., Takagi, A., and Koga, Y.: The effect of sofalcone on indomethacininduced gastric ulcers in a Helicobacter pylori-induced gnobiotic murine model. Aliment. Pharmacol. Ther., 14, 223-229, 2000.

[11] Oh, T.Y., Lee, J.S., Ahn, B.O., Cho, H., Kim, Y.B., Surh, Y.J.,
Cho, S.W., Lee, K.M., and Hahm, K.B.: Oxidative stress is more important than acid in the pathogenesis of reflux esophagitis in rats. Gut, 49, 364-371, 2001.

[12] Pai, R., Ohta, M., Itoni, R.M., Sarfeh, I.J., and Tarnawski, A.S.: Induction of mitogen-activated protein kinase signal transduction pathway during gastric ulcer healing in rats. Gastroenterology, 114, 706-713, 1998.

[13] Tetreault, M.P., Chailler, P., Rivard, N., and Menard, D.: Differential growth factor induction and modulation of human gastric epithelial regeneration. Exp. Cell Res., 306, 285-297, 2005.

[14] Tanigawa, T., Pai, R., Arakawa, T., Higuchi, K., and Tarnawski, A.S.: TGF- $\beta$ signaling pathway: its role in gastrointestinal pathophysiology and modulation of ulcer healing. J. Physiol. Pharmacol., 56, 3-13, 2005.

[15] Harsch, I.A., Brzozowski, T., Bazela, K., Konturek, S.J., Kukharsky, V., Pawlik, T., Pawlowski, E., Hahn, E.G., and Konturek, P.C.: Impaired gastric ulcer healing in diabetic rats; role of heat shock protein; growth factor, prostaglandins and proinflammatory cytokines. Eur. J. Pharmacal., 481, 249-260, 2003.

[16] Pai, R. and Tarnawski, A.S.: Signal transduction cascades triggered by EGF receptor activation: Relevance to gastric injury repair and ulcer healing. Dig. Dis. Sci., 43, 14S-22S, 1998.

[17] Arakawa, T., Watanabe, T., Fukuda, T., Higuchi, K., Fujiwara, Y., Kobayashi, K., and Tarnawski, A.S.: Ulcer recurrence: cytokine and inflammatory response-dependent process. Dig. Dis. Sci., 43, 61S-66S, 1998.

[18] Arakawa, T., Higuchi, K., Takashi, F., Fujiwara, Y., Kobayashi, K., and Kuroki, T.: Prostaglandins in the stomach; an update. J. Clin. Gastroenterol., 27, S1-S11, 1998.

[19] Arakawa, T., Nebiki, H., Uchida, T., Kimura, S., Higuchi, K., and Kobayashi, K.: Clinical assessment of quality of ulcer healing a new approach of endoscopic evaluation. Eur. J. Gastroenterol. Hepatol., 5, S87-S92, 1993.

[20] Arakawa, T. and Kobayashi, K.: Quality of ulcer healing a new concept to rank healed peptic ulcers. Gastroenterol. Jpn., 28, 158S-162S, 1993.

[21] Oh, T.Y., Shin, C.Y., Sohn, Y.S., Kim, D.H., Ahn, B.O., Lee, E.B., and Park, C.H.: Therapeutic effect of DA-9601 on chronic reflux gastritis induced by sodium taurocholate in rats. World J. Gastroenterol., 11, 7430-7435, 2005.

[22] Oh, T.Y., Ahn, G.J., Choi, S.M., Ahn, B.O., and Kim, W.B.: Increased susceptibility of ethanol-treated gastric mucosa to naproxen and its inhibition by DA-9601, an Artemisia asiatica extract. World J. Gastroenterol., 11, 7450-7556, 2005.

[23] Lee, S., Park, H.H., Son, H.Y., Ha, J.H., Lee, M.G., Oh, T.Y., Sohn, D.H., Jeong, T.C., Lee, S.H., Son, J.K., Lee, S.G., Jun, C.D., and Kim, S.H.: DA-9601 inhibits activation of the human mast cell line HMC-1 through inhibition of NF-кB. Cell Biol. Toxicol., 23, 105-112, 2007.

[24] Ahn, B.O., Ko, K.H., Oh, T.Y., Cho, H., Kim, W.B., Lee, K.J., Cho, S.W., and Hahm, K.B.: Efficacy of use of colonoscopy in dextran sulfate sodium induced ulcerative colitis in rats; the evaluation of the effects of antioxidant by colonoscopy. Int. J. Colorectal Dis., 16, 174-181, 2001. 
[25] Miyake, T., Suzaki, T., and Oishi, M.: Correlation of gastric ulcer healing features by endoscopy, stereoscopic microscopy and histology and a reclassification of the epithelial regenerative process. Dig. Dis. Sci., 25, 8-14, 1980.

[26] Watanabe, T., Arakawa, T., Fukuda, T., Higuchi, K., and Kobayashi, K.: Role of neutrophils in a rat model of gastric ulcer recurrence caused by interleukin $1 \beta$. Am. J. Pathol., 150, 971-979, 1997.

[27] Nagaike, R., Sawaguchi, A., Kawano, J., Aoyama, F., Oinuma, T., and Suganuma, T.: Regeneration of gastric mucosa during ulcer healing follows pathways that correspond to the ontogenetic course of rat fundic glands. Virchows Arch., 445, 580-588, 2004.

[28] Shahin, M., Konturek, J.W., Pohle, T., Schuppan, D., Herbst, H., and Domschke, W.: Remodeling of extracellular matrix in gastric ulceration. Microsc. Res. Tech., 53, 396-408, 2001.

[29] Longman, R.J., Douth Waite, J., Sylvester, P.A., Poulsom, R., Corfield, A.P., Thomas, M.G., and Wright, N.A.: Coordinated localization of mucins and trefoil peptides in the ulcer associated cell lineage and the gastrointestinal mucosa. Gut, 47, 792-800, 2000.

[30] Ribieras, S., Tomasetto, C., and Rio, M.C.: The pS2/TFF 1 trefoid factor, from basic research to clinical application. Biochim. Biophys. Acta., 1378, 61-77, 1998.

[31] Newton, J.L., Allen, A., Westley, B.R., and May, F.E.: The human trefoil peptide, TFF 1 is present in different molecular forms that are intimately associated with mucus in normal stomach. Gut, 46, 312-320, 2000.

[32] Watanabe, T., Higuchi, K., Tominaga, K., Fujiwara, Y., and Arakawa, T.: Acid regulates inflammatory response in a rat model of induction of gastric ulcer recurrence by interleukin
13. Gut, 48, 774-781, 2001.

[33] Ebert, M.P.A., Schafer, C., Chen, J., Hoffmann, J., Gu, P., Kubisch, C., Carl-McGrath, S., Treiber, G., Malfertheiner, P., and Rocken, C.: Protective role of heat shock protein 27 in gastric mucosal injury. J. Pathol., 207, 177-184, 2005.

[34] Tsukimi, Y. and Okabe, S.: Recent advances in gastrointestinal pathophysiology role of heat shock protein in mucosal defense and ulcer healing. Biol. Pharm. Bull., 24, 19, 2001.

[35] Halter, F., Tarnawski, A.S., Schmassmann, A., and Peskar, B.M.: Cyclooxygenase 2-implications on maintenance of gastric mucosal integrity and ulcer healing controversial issues and perspectives. Gut, 49, 443-453, 2001.

[36] Kimura, A., Tsuji, S., Tsuji, M., Sawaoka, H., Iijima, H., Kawai, N., Yasumaru, M., Kakiuchi, Y., Okuda, Y., Ali, Z., Nishimura, Y., Sasaki, Y., Kawano, S., and Hori, S.: Expression of cyclooxygenase-2 and nitrotyrosine in human gastric mucosa before and after Helicobacter pylolri eradication. Prostaglandins Leukotrienes Essential Fatty Acids, 63, 315322, 2002.

[37] Halliwell, B.: What nitrates tyrosine? Is nitrotyrosine specific as biomarker of peroxynitrite formation in vivo. FEBS Lett., 411, 157-160, 1997.

[38] Ernst, H., Grunert, S., Schneider, H.T., Beck, W.S., Brune, K., and Hahn, E.G.: Distribution of extra cellular matrix proteins in indomethacin-induced lesions in the rat stomach. Scand. J. Gastroenterol., 30, 847-853, 1995.

[39] Wahl, S.M. and Wang, H.: Mccartney-Francis N. Role of growth factors in inflammation and repair. J. Cell. Biochem., 40, 193-199, 1989. 